\title{
Exenatid ist kardiovaskulär sicher, aber ohne kardioprotektiven Nutzen
}

\author{
Das GLP1-Analogon Exenatid hat in der EXSCEL-Studie die Erwartungen nur partiell erfüllt: Zwar \\ erwies sich das Antidiabetikum in kardiovaskulärer Hinsicht als sicher - zur erhofften Reduktion \\ von Ereignissen wie Herzinfarkt und Schlaganfall kam es - anders als in Studien mit Liraglutid \\ und Semaglutid - jedoch nicht.
}

\begin{abstract}
Die Ergebnisse der Phase-IIIb/IV-Studie EXSCEL (EXenatide Study of Cardiovascular Event Lowering) sind aktuell auf dem europäischen Diabetes-Kongress (EASD) in Lissabon vorgestellt und simultan im „New England Journal of Medicine“ publiziert worden. Danach sind die gesteckte Zielen der Studie nur zur Hälfte erreicht worden. In der Studie ging es darum, sowohl die kardiovaskuläre Wirksamkeit als auch Sicherheit des GLP-Analogons Exenatid in der langwirksamen Formulierung (einmal wöchentliche Injektion) im Vergleich zu Placebo unter Beweis zu stellen. Dies sollte anhand eines primären kombinierten Studienendpunkts erfolgen, der sich aus den Ereignissen kardiovaskulär bedingter Tod, Myokardinfarkt und Schlaganfall zusammensetzte.
\end{abstract}

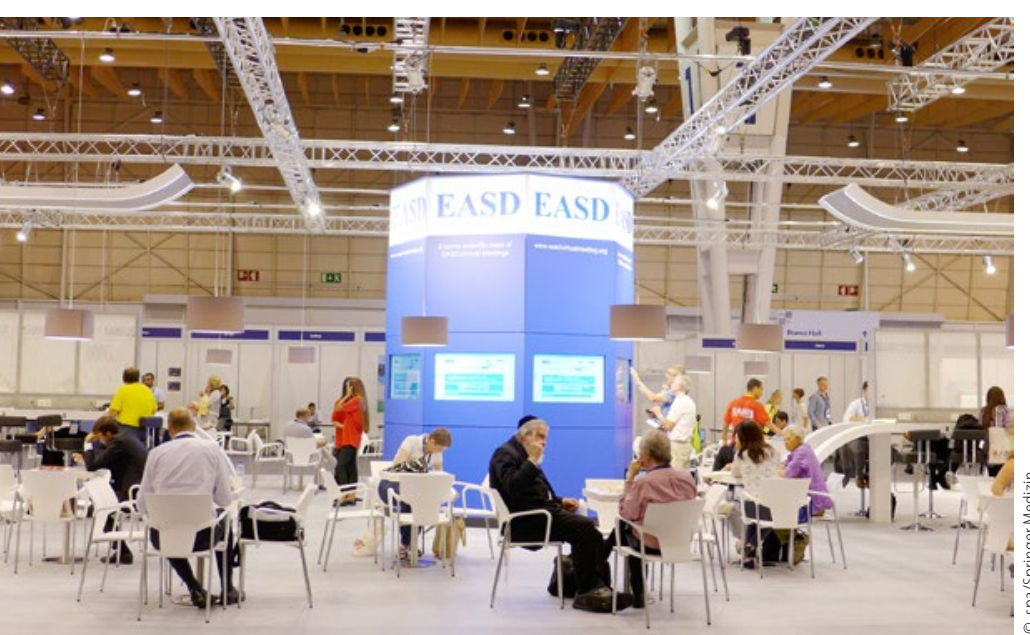

Ausruhen, planen, austauschen - wem die Sonne draußen zu grell war, der ließ sich in den Hallen des EASD-Kongresses nieder.

\section{Sicherheit belegt, Wirksamkeitsnachweis verfehlt}

Die gute Nachricht: In puncto kardiovaskuläre Sicherheit konnte die „Nicht-Unterlegenheit“ von Exenatid im Vergleich zu Placebo nachgewiesen werden ( $p<0,001$ für Nicht-Unterlegenheit). Die weniger gute Nachricht: Das Ziel, auch einen klinischen Vorteil von Exenatid in Form einer signifikanten Reduktion von kardiovaskulären Ereignissen zu dokumentieren, wurde verfehlt. Nach Ablauf von 3,2 Jahren (mediane Beobachtungsdauer) betrug die Rate für die primären Endpunktereignisse 11,4\% in der Exenatid-Gruppe und 12,2\% in der Placebo-Gruppe (Hazard Ratio 0,91). Der Unterschied zugunsten von Exenatid war zu klein, um statistisch als Ausdruck von Überlegenheit gelten zu können ( $\mathrm{p}=0.06$ für Überlegenheit). Weder bei der kardiovaskulären Mortalität ( $4,6 \%$ vs. $5,2 \%$ ) noch bei Myokardinfarkten $(6,6 \%$ vs. $6,7 \%)$ oder Schlaganfällen $(2,5 \%$ vs. $2,9 \%)$ gab es signifikante Unterschiede zwischen Exenatid und Placebo. Auch im Hinblick auf mögliche schwere Komplikationen wie akute Pankreatitis oder Pankreaskarzinom unterschieden sich beide Gruppen nicht signifikant.

\section{Mögliche Gründe für die diskrepanten Ergebnisse}

Wo liegen die Gründe, dass in EXSCEL - anders als in der LEADER-Studie (mit Liraglutid) und der SUSTAIN-Studie (mit Semaglutid) - keine günstige Wirkung des GLP1-Rezeptoragonisten auf das kardiovaskuläre Risiko zum Vorschein gekommen ist. Die Studienautoren spekulieren, dass ein Grund die im Vergleich zu LEADER kürzere Beobachtungsdauer (3,2 vs. 3,8 Jahre) und Behandlungsdauer (2,4 vs. 3,5 Jahre) gewesen sein könnte. Sie verweisen auch auf den im Schnitt niedrigeren $\mathrm{HbA}_{1 c^{-}}$ Werte der EXSCEL-Teilnehmer (8,0\% vs. 8,7\%). Zudem sei die Rate an Therapieabbrüchen unter Exenatid höher gewesen als unter Placebo. Auch schließen sie nicht aus, dass in der Placebo-Gruppe mehr Patienten als Teil ihrer antidiabetischen Therapie Substanzen wie SGLT2-Hemmer erhalten haben, die ebenfalls das kardiovaskuläre Risiko verringern und so den Unterschied zwischen beiden Gruppen womöglich minimiert haben.

\section{Niedrigere Rate für Gesamtmortalität}

Abgesehen von der fehlenden Signifikanz sehen die EXSCEL-Autoren ihre Ergebnisse mehr oder weniger in Übereinstimmung mit denen der anderen Studien zur Frage der kardiovaskulären Sicherheit und Wirksamkeit von GLP1-Rezeptoragonisten. So gab es auch bei der Gesamtmortalität (6,9\% vs. 7,9\%) in EXSCEL einen Unterschied zugunsten von Exenatid, der sogar nominell signifikant war. Aufgrund des hierarchischen Testplans der Studie könne dieser Unterschied aber nicht als statistisch signifikant betrachtet werden, schränken die Studienautoren ein.

In die EXSCEL-Studie sind weltweit 14.752 Patienten mit Typ2-Diabetes aufgenommen worden, von denen die meisten $(73,1 \%)$ bereits eine kardiovaskuläre Erkrankung aufwiesen. Sie sind auf zwei Gruppen randomisiert und entweder mit Exenatid (2 mg subkutan einmal wöchentlich) oder Placebo zusätzlich zur üblichen Standardtherapie behandelt worden.

Peter Overbeck

Quelle: Vorgestellt beim 47. EASD Annual Meeting, 11. - 15. September 2017, Lissabon; Holman R.R. et al.: Effects of Once-Weekly Exenatide on Cardiovascular Outcomes in Type 2 Diabetes, N Engl J Med 2017, online 14. September 2017. DOI: 10.1056/NEJMoa1612917 